\title{
IMPACT ASSESSMENT OF EXTREME STORM EVENTS USING A BAYESIAN NETWORK
}

\author{
C.(Kees) den Heijer ${ }^{1,2}$, Dirk T.J.A. Knipping ${ }^{3}$, Nathaniel G. Plant ${ }^{4}$, Jaap S.M. van Thiel de Vries ${ }^{2,1}$, \\ Fedor Baart ${ }^{2,1}$, Pieter H.A.J.M. van Gelder ${ }^{1}$
}

\begin{abstract}
This paper describes an investigation on the usefulness of Bayesian Networks in the safety assessment of dune coasts. A network has been created that predicts the erosion volume based on hydraulic boundary conditions and a number of cross-shore profile indicators. Field measurement data along a large part of the Dutch coast has been used to train the network. Corresponding storm impact on the dunes was calculated with an empirical dune erosion model named Duros + . Comparison between the Bayesian Network predictions and the original Duros+ results, here considered as observations, results in a skill up to 0.88 , provided that the training data covers the range of predictions. Hence, the predictions from a deterministic model (DURos+) can be captured in a probabilistic model (Bayesian Network) such that both the process knowledge and uncertainties can be included in impact and vulnerability assessments.
\end{abstract}

Keywords: Dune erosion; Extreme conditions; probabilistic approach; Bayesian Network model

\section{INTRODUCTION}

Large parts of The Netherlands are below mean sea level and are protected from flooding by coastal dunes. Extreme waves and water levels during severe storms may cause failure of the dunes as a sea defence. Consequently, serious damage due to flooding and direct wave attack could occur, resulting in loss of life and property. Successful coastal management requires that assessments of coastal safety are updated using accurate information and robust methodologies. In addition, reinforcement measures will be taken if the actual safety level does not meet an appropriate agreed standard. The agreed Dutch safety standard is largely based on an economic decision (Van Dantzig, 1956), being a trade off between reinforcement cost of the flood protection system and risk of damage (Vrijling, 2001). Due to the high population density and the high economical value in low lying (below mean sea level) areas of The Netherlands, being potentially threatened by flooding, small probabilities of failure are accepted in the safety standard. Small failure probabilities correspond to extreme design conditions. Extreme design conditions imply, given a relatively short period of available measurements, a lack of (local) field observations with the magnitude of interest. Therefore, data extrapolation and numerical model simulations are key items in the current safety assessment method. As a consequence, uncertainties play an important role.

The dune safety assessment approach, presently used for the Dutch coast, is based on an erosion profile model (DuRos+; Van Gent et al., 2008) and the probabilistic FORM (Hasofer and Lind, 1974) and Monte Carlo (Fishman, 1996) methods. The dune erosion model in this approach is computationally cheap, but has a limited applicability due to its underlying assumptions (Den Heijer et al., 2011). The model assumes a sandy coast (no hard elements) that is uniform in alongshore direction (no curved coastline) and attacked by perpendicular incoming waves. As an alternative, the 2DH XBeach model (Roelvink et al., 2009) is proposed, for the areas where the assumptions are not met. The latter model shows results with significant predictive skill under storm conditions (McCall et al., 2010), but is computationally much more expensive, and therefore less suitable for application in combination with the above mentioned probabilistic methods.

The uncertainties in the safety assessment can be reduced by (1) extending the time series of local measurements, (2) improving the numerical models and (3) utilizing remote measurements. Longer time series of local measurements improve the probability distributions of the forcing variables and might include response data of extreme events. However, the probability of capturing a relevant extreme event is small and the value of the measurement data only improves slowly over time. Improving the numerical models enhances the relation between forcing variables and response indicators. Utilizing remote measurements can provide data with high magnitude at higher frequencies, compared to the local data.

A Bayesian Network is a probabilistic graphical model that represents a set of random variables and their conditional dependencies via a directed acyclic graph (DAG). It provides information about the probability of an event, and that probability can be updated by adding additional evidence. Plant and Holland (2011a,b) successfully demonstrated the use of a Bayesian Network model for prediction and assimilation of processes in the surf zone. A Bayesian Network relies on data, so-called training

\footnotetext{
${ }^{1}$ Delft University of Technology, Delft, The Netherlands

${ }^{2}$ Deltares, Delft, The Netherlands

${ }^{3}$ Van Oord, Rotterdam, The Netherlands

${ }^{4}$ U.S. Geological Survey, St. Petersburg, FL, USA
} 
data, and is capable of combining different data sources, such as local and remote field measurements, laboratory data and model simulations. Due to the modular nature of such a network, it is a useful framework to investigate the consequences of 'new' variables or processes. In addition, the network can capture results of computationally expensive numerical model simulations and provide instantly predictions that include those results. In this way, the use of computationally expensive process-based models for complex areas in a probabilistic context becomes practically feasible.

To effectively benefit from the advantages of Bayesian Networks in dune safety assessment practice, a number of steps need to be taken. First of all, it needs be investigated to what extent the Bayesian Network is capable of reproducing the underlying empirical and/or numerical model results. Secondly, the Bayesian Network approach should be compared to the current Dutch safety assessment, the latter being a combination of an empirical dune erosion with the probabilistic FORM or Monte Carlo method. Finally, the usefulness of a Bayesian Network for the safety evaluation of complex areas, such as curved coastlines, should be demonstrated.

The aim of this paper is to investigate whether a Bayesian Network can reproduce the dune erosion volumes as calculated by the empirical dune erosion model Duros+. To that end, a network is constructed that includes the input variables of Duros + and predicts the dune erosion volume. The forcing data that is provided to the Bayesian Network is obtained from available probability distributions and the erosion volumes are based on DURos+ calculations.

\section{BAYESIAN NETWORKS}

A Bayesian Network is one example in the family of probabilistic graphical models. Probabilistic graphical models combine probability theory and graph theory. The definition of a probabilistic model can be found in (Pearl, 1988): "A set $U$ of discrete random variables together with a joint probability distribution $P(U)$ defined over these variables." A probabilistic graphical model provides a natural tool for dealing with two problems occurring in engineering: uncertainty and complexity. Fundamental in the idea of a graphical model is the notion of modularity; a complex system is built by combining simpler parts. The probability theory provides the glue whereby the parts are combined, ensuring that the system as a whole is consistent, and providing ways to interface models to data. Graphical models provide an intuitively appealing interface by which the user can model highly interacting sets of variables.

Once a Bayesian Network is constructed it can be used for several applications:

1. Posterior probabilities: probability of an event given any evidence

2. Most likely explanation: scenario that explains evidence

3. Rational decision making: maximize expected utility

4. Effect of intervention: causal analysis

\section{Difference between Bayesian Network and other probabilistic graphical models}

To put the Bayesian Network in the context of other probabilistic graphical models the main differences between the Bayesian Network and two other often used models will briefly be discussed below.

- Markov model or hidden Markov model: also a graphical probabilistic model comparable to a Bayesian Network. However, a Markov net is capable to represent cyclic dependencies, which allows to explore feedback mechanisms. In a regular Markov model, the state is directly visible to the observer, and therefore the state transition probabilities are the only parameters. In a hidden Markov model, the state is not directly visible, but output, dependent on the state, is visible. The main difference between a Markov model and a Bayesian Network is that a Markov model concept does not have history (i.e., prior information).

- Neural Network: An (artificial) neural network is a system based on the operation of biological neural networks, in other words, it is an emulation of biological neural system. Often people refer to Neural networks as a 'black box' due to the difficulty in understanding the decision making process of the Neural network. A Neural network contains by default continuous variables which are basically all connected with each other. The strength of the relations between all pairs of variables is based on the correlation in the underlying data. In contrast, a Bayesian Network has by default discrete variables and the dependencies (connections) have to be chosen manually, which allows for the inclusion of system knowledge.

There are more graphical models (e.g. restricted Bollzman machine, junction (or clique) tree) but they are less applied in probabilistic theory and are not be discussed here. 


\begin{tabular}{ll|ll}
$\mathrm{E}$ & $\mathrm{B}$ & \multicolumn{2}{|c}{$P(A \mid E, B)$} \\
\hline $\mathrm{e}$ & $\mathrm{b}$ & $P(A \mid e, b)$ & $P(\neg A \mid e, b)$ \\
$\mathrm{e}$ & $\neg \mathrm{b}$ & $P(A \mid e, \neg b)$ & $P(\neg A \mid e, \neg b)$ \\
$\neg \mathrm{e}$ & $\mathrm{b}$ & $P(A \mid \neg e, b)$ & $P(\neg A \mid \neg e, b)$ \\
$\neg \mathrm{e}$ & $\neg \mathrm{b}$ & $P(A \mid \neg e, \neg b)$ & $P(\neg A \mid \neg e, \neg b)$
\end{tabular}

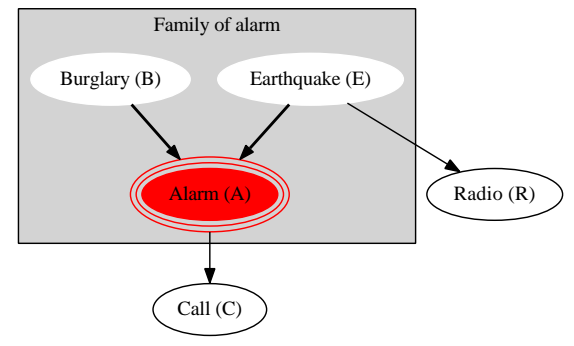

Figure 1: Example of a Bayesian Network considering an alarm that can can be triggered by either an earthquake or a burglar. Left: the conditional probability table for the node 'alarm', $\neg$ means 'not', so $\neg b$ means no burglary. Right: Bayesian Network of the situation with in the box the family alarm.

A Bayesian Network is characterized by:

1. Modular representation of knowledge. A complex system is built by combining simpler parts. What is known and how are the variables mutual correlated.

2. Local, distributed algorithms for inference and learning. Lower time complexity (less time for inference)

3. Intuitive (possible causal) interpretation.

4. Factored representation may have exponentially fewer parameters than full joint representation (remember the burglar alarm example) Lower sample complexity (less data for learning).

\section{Bayesian Network approach}

A Bayesian Network is a method of reasoning using probabilities ${ }^{5}$, where the nodes represent variables and arrows represent direct influence between the nodes. Nodes can represent any kind of variable: a measured parameter, a latent (hidden) variable or a hypothesis. The graph itself is a directed acyclic graph (DAG) which means that arrows have a direction (they point from one node to another) and there are no cycles or loops in the network (to prevent new information from being affected by its own result).

If there is an arrow from node $A$ to another node $B, A$ is called a parent of $B$, and $B$ is a child of $A$. In addition, the parents of $A$ (and further upstream) are the ancestors of $B$. The set of parent nodes of a node $x_{i}$ is denoted by parent $_{x_{i}}$. In a Bayesian Network the joint probability distribution of the node variables can be written as the product of the local distributions of each node and its parents as

$$
P\left(x_{i}, \ldots, x_{n}\right)=\prod_{i=1}^{n} P\left(x_{i} \mid \text { parent } s_{x_{i}}\right)
$$

In this sense Bayesian Networks have built-in independence assumptions. In summary (Wikle and Berliner, 2007): one has prior belief (information) in a certain event, collects data, and then updates that belief given that new data (information). In the Bayesian world, the definition of belief is simply the conditional probability given the evidence. So, the task of the Bayesian Network is to compute the posterior distribution function.

Since Bayesian Networks are not widely used in coastal engineering practice, we illustrate illustrate the principles with an example. Here, the burglar-earthquake alarm example (adopted from Pearl, 1988) will be used, depicted in Figure 1. This network represents a distribution on five variables $P(B, E, A, C, R)$. The intuition behind the model is that either burglaries or earthquakes (Pearl lived in Los Angeles) can trigger an alarm. If the alarm rings your neighbour may call you at work to let you know. When on your rush way home you hear a radio report of an earthquake, the degree of confidence (i.e. belief) that there was a burglary will diminish. This problem is captured in Figure 1. Nodes and edges form the qualitative part, the quantitative part of the network consist out of a set of conditional probability distributions. The table in Figure 1 shows the conditional probability for the node 'alarm' Together both parts form a unique distribution in factored form (see also Equation 1):

$$
P(B, E, A, C, R)=P(B) P(E) P(A \mid E, B) P(R \mid E) P(C \mid A)
$$

Via the conditional probability definition (Equation 1) one can see that a node is independent of its ancestors given its parents. In this example this means: the node 'call' (child) is independent of

\footnotetext{
${ }^{5}$ Several names are used to describe the method of reasoning using probabilities, such as belief networks, Bayesian Networks, knowledge maps, probabilistic and causal networks. In this work Pearl's name, Bayesian Networks (Pearl, 1988), is adopted.
} 
'burglary' and 'earthquake' (ancestors) given the node 'alarm' (parent). So, given 'alarm', 'call' is conditionally independent of 'burglary' and 'earthquake'. In Bayesian terms this is called, the nodes 'burglary' and 'call' are $d$-seperated. There is no direct connecting path between both nodes. Two nodes are d-connected if either there is a causal path between them, or there is evidence that renders the two nodes correlated to each other (Charniak, 1991). Together they form a 'family'. In Figure 1 the family of the node 'alarm' is highlighted in the grey box.

Equation 1, expressed in Equation 2 for our burglar-earthquake alarm example, specifies the required parameters for this Bayesian Network, the probability of every node given all possible combinations of its parents. Representing joint probability distributions via conditional independence is very useful, it reduce the dimensionality of the network from full joint probability table, $2^{5}-1=31$ to $1+1+4+2+2=10$ parameters (Equation 2). This savings might not seem great, but if the net is doubled in size the number of parameters become $2^{10}-1=1023$. One can imagine that in case of larger nets and non-boolean nodes (not just two possible classes, non-dichotomous) the number of parameters will grow exponentially.

\section{Bayesian inference}

Bayesian inference is the application of Bayes' theorem to calculate how the degree of belief in a proposition changes due to evidence. For illustration, let $X$ denote unobservable quantities of interest and $Y$ the data. The full probability model can always be factored in components $p(x, y)=p(y \mid x) p(x)=$ $p(x \mid y) p(y)$. Note that $p(x, y)$ means the probability of $x$ and $y$. Applying Bayes' rule gives:

$$
p(x, y)=\frac{p(y \mid x) p(x)}{p(y)} \quad \text { provided that } \quad 0<p(y)<\infty
$$

It is good to examine each component of Bayes' rule separately (Wikle and Berliner, 2007).

- Data distribution, $p(y \mid x)$

Distribution of the data, given the unobservables. When viewed as a function of $X$ for fixed $y$, it is known as a likelihood function, $L(x \mid y)$. A key is that one thinks of the data conditioned on $x$. For example, if $Y$ represents imperfect observations of temperature, and $X$ is the true temperature, then $p(x \mid y)$ quantifies the distribution of measurement errors in observing temperature, reflecting instrument error as well as possible biases.

- Prior distribution, $p(x)$

This distribution quantifies the a priori (beforehand) understanding of the unobservable quantity of interest. For example, if $X$ corresponds to a temperature, then one might base this prior distribution on historical information (climatology). A choice of a prior distribution can be subjective and forms a integral part of the Bayesian inference.

- Marginal distribution, $p(y)=\int p(y \mid x) p(x) d x$

For the observations $Y, p(y)$ can be thought of as the 'normalizing constant' in Bayes' rule. Unfortunately, it is only for specific choices of the data and prior distribution that one can solve this integral analytically (Cooper, 1990).

- Posterior distribution, $p(x \mid y)$

This distribution of the unobservables given the data is for this paper the primary interest for inference. The posterior is the update of our prior knowledge about $x$ given the actual observations $y$.

\section{APPROACH}

This paper describes an initial step in an attempt to utilize Bayesian Networks to be able to perform a proper dune safety assessment in complex coastal areas, such as curved coastlines. In this paper a network is presented that aims to reproduce the by DuRos+ calculated dune erosion volumes. Therefore, the nodes and the relations in the network are based on the variables of the dune erosion model.

Four steps need to be taken to come to a useful Bayesian Network model: data gathering, network construction, training and validity check. The steps are interlinked with each other, because the required data depends on the nodes in the network. In addition, depending on the results of the validity check, the steps may be repeated in order to reach satisfactory results. The next section provides more detailed information about the steps and the related model choices.

\section{MODEL SETUP}

For this research, Dutch cross-shore transects of bed levels together with corresponding offshore wavebuoy data and grain size, serve as input for the Bayesian Network model. The Bayesian Network 


\begin{tabular}{lll} 
Type & Data & Source \\
\hline measured & profile information & JARKUS \\
extrapolated & hydraulic boundary conditions & WL | Delft Hydraulics (2007) \\
modelled & coastal response to a storm & DURos+ (Van Gent et al., 2008)
\end{tabular}

Table 1: Types of data that serve as input for the Bayesian Network.

\begin{tabular}{llll} 
Variable & Mean & Standard Deviation & Distribution type \\
\hline Water level $(W L)$ & $f\left(P_{\text {exc }}\right)$ Figure 4 & - & Conditional Weibull \\
Wave height $\left(H_{s}\right)$ & $f(h)$ Figure 5 & $0.6 \mathrm{~m}$ & Normal \\
Wave period $\left(T_{p}\right)$ & $f\left(H_{s}\right)$ Figure 5 & $1 \mathrm{~s}$ & Normal \\
Grain size $\left(D_{50}\right)$ & $159 \mu m-277 \mu m$ & $8 \mu m-37 \mu m$ & Normal
\end{tabular}

Table 2: Probability distributions

model is developed to predict dune erosion volumes due to an extreme storm event described by its hydraulic boundary conditions and given the local cross-shore profile.

The data needed for the training of the Bayesian Network can be divided in three categories, specified in Table 1. Cross-shore profiles along the Dutch coast are available in the JARKUS dataset (yearly coastal bathymetry measurements). For generation of the data, cross-shore profiles at the same coastal areas as selected by Den Heijer et al. (2011) have been used (see Figure 2). The hydraulic boundary conditions have been sampled from probability distributions as obtained from WL | Delft Hydraulics (2007). The dune response data has been gathered based on simulations with Duros+ (Van Gent et al., 2008).

Figure 3 gives an overview of the Bayesian Network with the nodes (variables) and their relations (edges). The main goal variable is the erosion volume, which is dependent on several load and strength variables. The load variables are described by probability distributions. The probability distributions that were used for the development of the safety assessment method for the Dutch dune coast (WL | Delft Hydraulics, 2007) have been reused in this paper (Table 2). The strength variables are related to the coastal area and need to be derived from individual cross-shore profiles. Apart from the grain size (Table 2), four profile indicators are defined to characterize the cross-shore profile, illustrated in Figure 6:

1. Profile volume, $V_{\text {prof }}$

Profile volume enclosed by horizontal reference levels MSL $+3 \mathrm{~m}$ (dune foot) and MSL-4m, together with the vertical boundaries landward and seaward of the intersection of the horizontal reference levels with the initial bottom profile. (Green box in Figure 6) This area indicates the potential accretion volume and is independent of dune height.

2. Slope of $V_{\text {prof }}, s_{\text {vol }}$

Slope of the volume as indicated in profile indicator 1 (purple line). This slope is comparable to the foreshore slope and is added to give more information of the shape of the profile volume.

3. Beach width, $W_{b}$

Beach width is the cross-shore distance crest of the first dune row and the shoreline. So, in fact here the beach width represents the regular beach width extended with half of the first dune row width.

4. Maximum crest level of first dune row, $z_{c}$

Maximum crest level of the first dune row. The dune height is an important indicator of the strength of a dune, since it gives information about the dry volume of sand.

The network needs to be trained, after the data gathering and the construction of the network. Training means the establishment of the conditional probabilities of all the nodes based on the available data. It is important to realize that the predictions cannot go beyond the data ranges of the nodes and also cannot cover combinations that are not in the dataset. In that respect, the difference between the Holland coast and the Wadden area is relevant. The Holland coast is a mainly closed and approximately straight without deep channels and with only limited variation in foreshore slope. The Wadden coast, on the contrary, consists of a series of barrier islands with very mild sloped areas but also deep channels at some locations.

Finally, validity check has to be done. The term validity, in this case, defines the degree to which the Bayesian Network can represent the Dutch coastal indicators. Here the test is designed to measure 


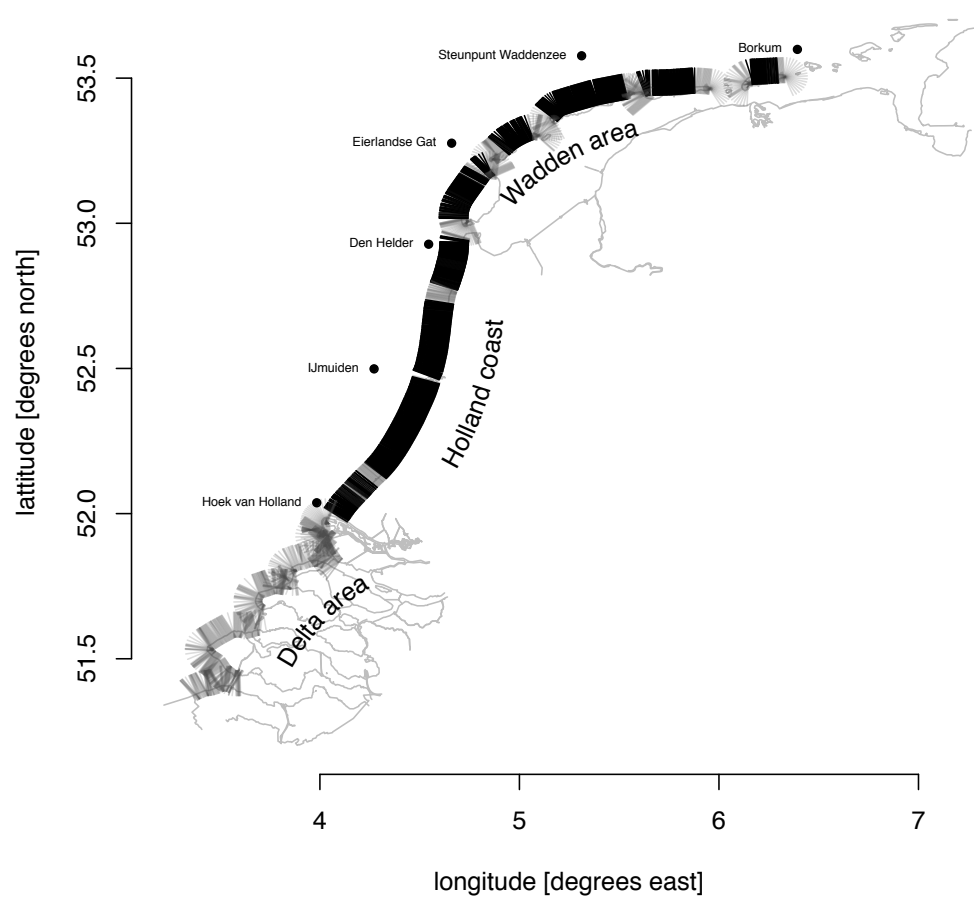

Figure 2: Overview of transects that were selected to create the dataset (black). The remaining, not selected, transects are in gray.

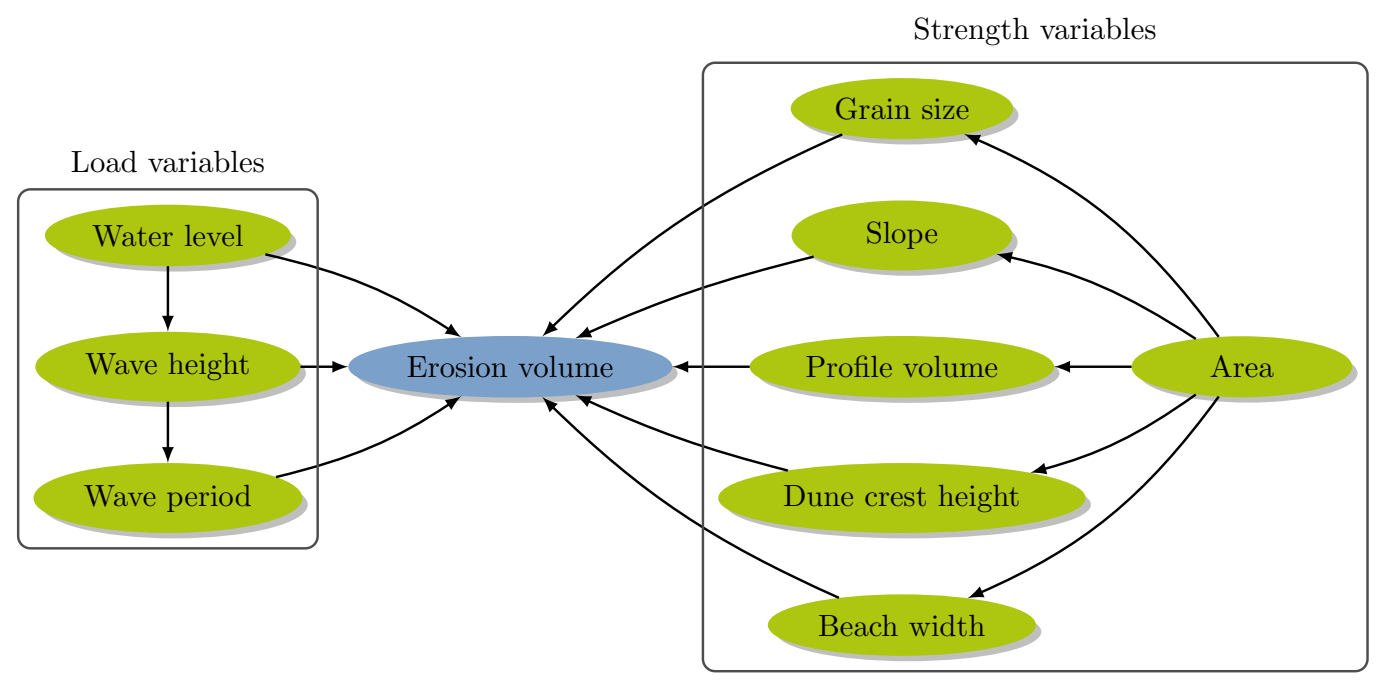

Figure 3: Overview of the Bayesian Network. 


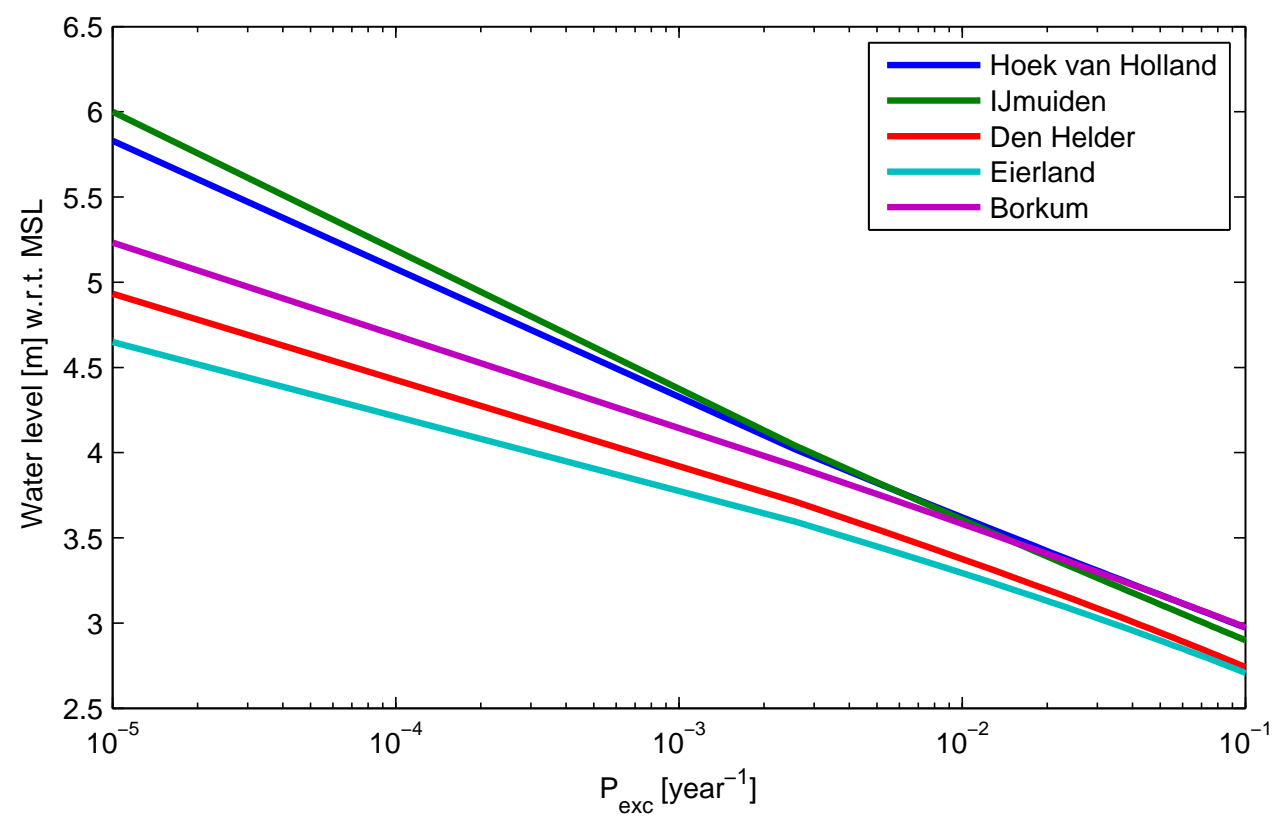

Figure 4: Water level as function of the exceedance probability (WL | Delft Hydraulics, 2007; Den Heijer et al., 2011).
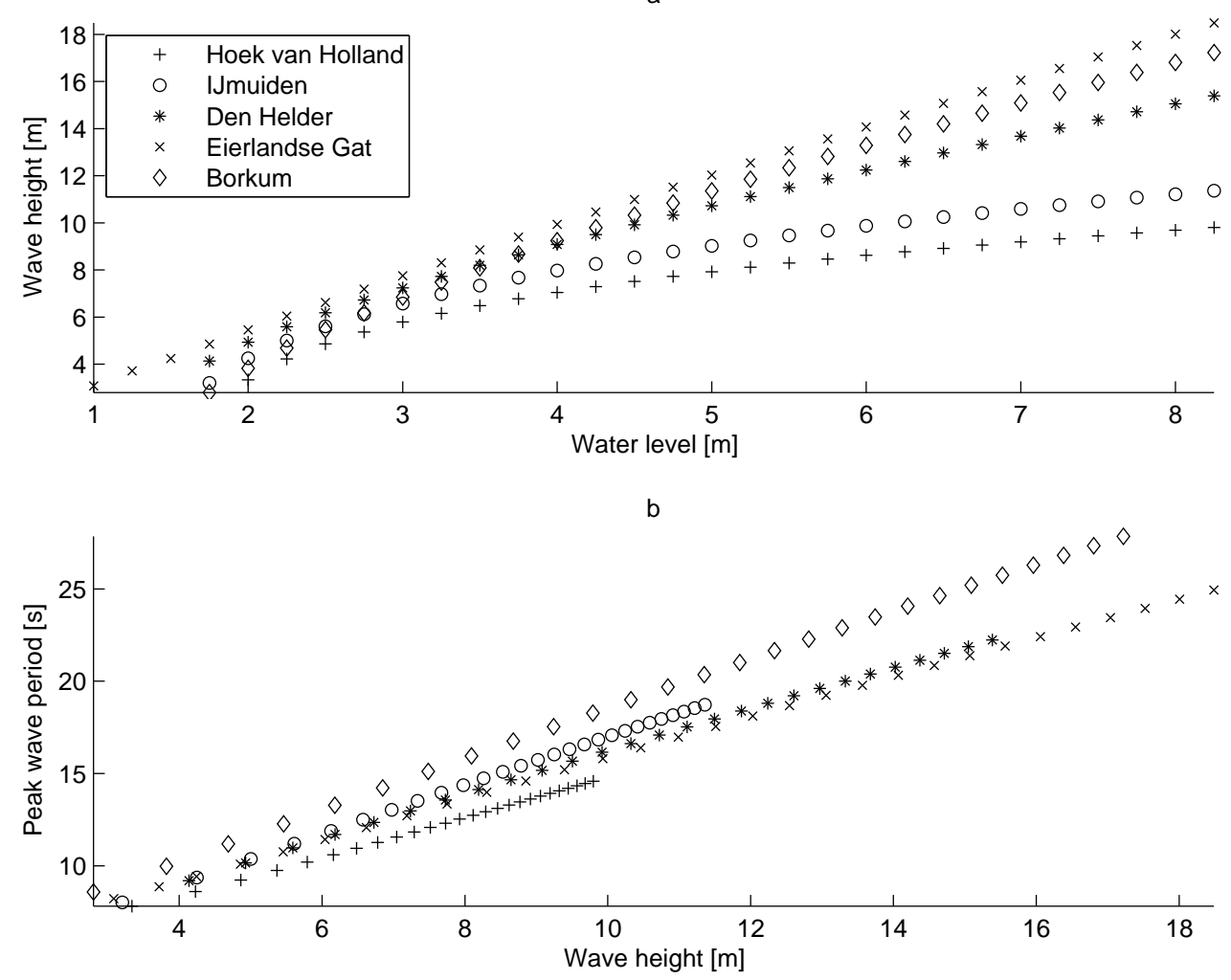

Figure 5: (a) Mean wave height as function of water level. (b) Mean peak wave period as function of wave height. 


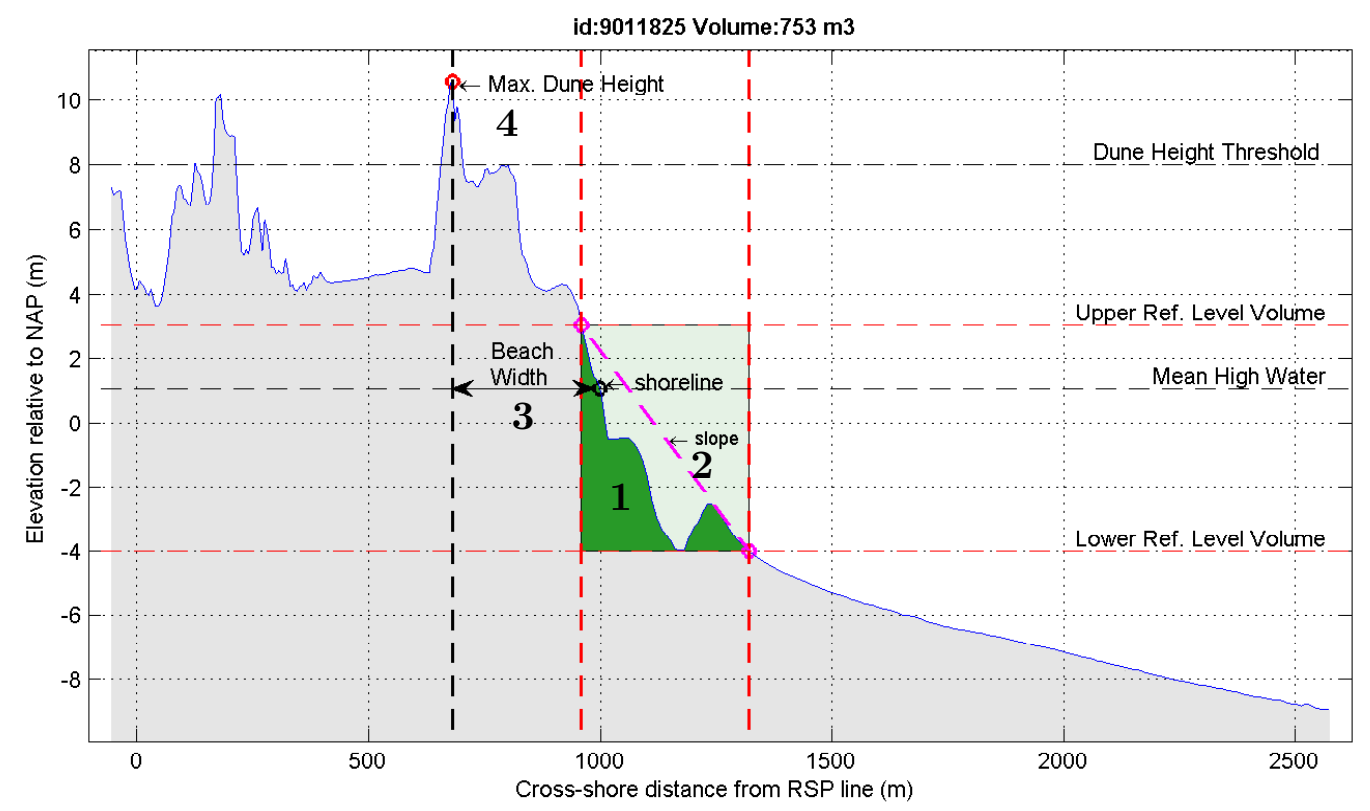

Figure 6: Profile indicators for a random selected pre-storm cross-shore profile. The numbers correspond to the numbers in the list of profile indicators.

dune erosion as result of a storm event. When the test appears reasonable on its face to model the real system, then the model has face validity. A test can be said to have face validity if it 'looks like' it is going to measure what it is supposed to measure. For instance one would expect (on average) a finer grain size at the Wadden area with respect to the Holland coast. In addition, one expects a high erosion volume accompanied by a high water level. If the Bayesian Network meets this expectations one can say the Bayesian Network has face validity.

Apart from the face validity, that can be considered as a first check, the prediction skill of the network needs to assessed in a hind cast. To that end, the predicted erosion volumes by the Bayesian Network are compared to the erosion volumes as calculated by DuRos + , considered as observations in this paper. To determine the degree to which the Bayesian Network can recover the simulated erosion volume (morphological change) and whether its uncertainty estimates are consistent with the actual prediction errors two evaluation methods will be used. First, the prediction skill will be quantified using a simple linear regression model relating predictions to the actual observations (in our case DURos+ calculations). Second, the prediction skill is quantified by evaluating the likelihood of each observation, as predicted, and comparing this likelihood to the prior likelihood. Both methods will be explained in detail below. The methods will be applied to hindcast predictions using the training dataset to update some of the variables (e.g. water levels, wave height, profile volume and dune crest).

A simple regression model is used to quantify the prediction skill relating predictions to the actual observations. Therefore we wish to find the equation of the straight line

$$
\hat{y}=\alpha+\beta \bar{x}
$$

Here, $\hat{y}$ is the regression estimate based on $\bar{x}$, the Bayesian-mean predicted value, and includes corrections for bias $\alpha$ and $\beta$. The Bayesian-mean value is

$$
\bar{x}_{i}=\sum_{j=1}^{J} p\left[\Delta D_{j} \mid \text { inputs }_{i}\right] \Delta D_{j},
$$

where the summation is over the $j=1,2, \ldots, J$ discrete bins from the prediction obtained for each case $i$. Here inputs $_{i}$ represents a 'case' (a particular combination of the possible input variables). The regression skill for this prediction is

$$
s=1-\frac{\sum_{i=1}^{I} \sigma_{x, i}^{2}\left[y_{i}-\hat{y}_{i}\right]^{2}}{\sum_{i=1}^{I} \sigma_{x, i}^{2}\left[y_{i}\right]^{2}},
$$



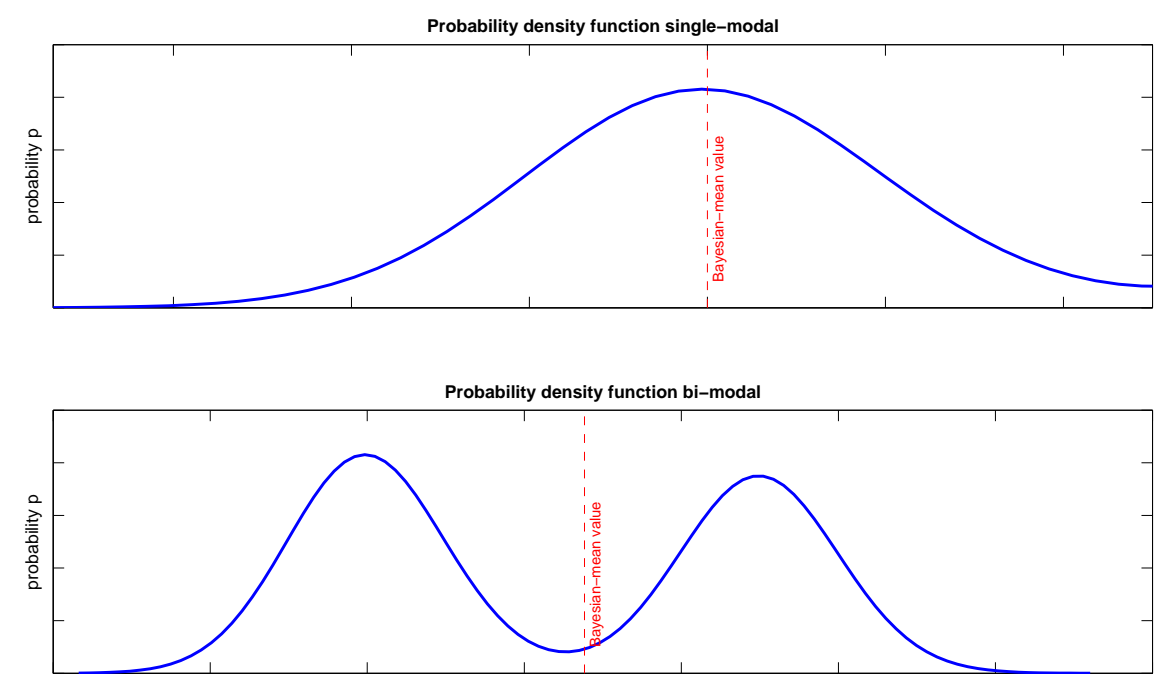

Figure 7: Example of two possible probability density distributions describing the result. In case of a modal distribution Bayesian-mean value is a good method to quantify the prediction skill but it gets bad when the distribution is more-or-less bi-modal.

where the summation is over all considered cases, $i=1,2, \ldots, I$. The measure skill describes a weighted percentage of observed variance that is explained by the Bayesian-mean prediction. The weighting factors are the prediction variance around the Bayesian-mean value and do not depend on the observations. The variances are computed as

$$
\sigma_{x, i}^{2}=\sum_{j=1}^{J} p\left[\Delta D_{j} \mid \text { inputs }_{i}\right]\left[\Delta D_{j}-\bar{x}_{i}\right]^{2} .
$$

The Bayesian-mean value represents a robust predicted value (Plant and Stockdon, 2012) and the variance of the prediction provides a measure of prediction uncertainty used as weighting term in the regression and skill estimates. However, the Bayesian mean value is strictly appropriate if the result is more-or-less normally distributed. When a result is bi-modal for instance (see Figure 7) the Bayesianmean value will fall between the two most likely outcomes and is a horrible prediction. Therefore, another method to quantify the prediction skill will be used as well.

\section{Log-likelihood ratio}

The log-likelihood ratio makes it possible to describe the skill of the updated probability distributions by evaluating the likelihood of each observation, and comparing this likelihood to the prior likelihood. Likelihood is a measure of how likely an event is. For instance for a forecast $F$ with given observables $O, p(F \mid O)$, the likelihood function is the probability of the observations if the forecast is known: $p(O \mid F)$. The likelihood term can include both model and observation errors. That is, if the model and measurements were error free, than an observation would be likely only if it equalled the forecast value. In reality, there are numerous errors causing spread in the likelihood function.

Here, with likelihood the maximum likelihood function is meant. This function describes the likelihood of a set of parameter values given some observed outcomes that is equal to the probability of those observed outcomes given those parameter values. Since, one is interested in the most likely parameters the likelihood is labelled as maximum likelihood. In this case the log-likelihood ratio will be used (Equation 8) to describe the maximum likelihood. A logarithmic function will be used to make the process of differentiating (to find the maximum likelihood) easier. Moreover, because the logarithm is an increasing function, the likelihood function and the log-likelihood function attain their extreme values for the same values.

$$
L=\log \left\{p\left[\Delta D_{j} \mid \text { inputs } s_{i}\right]_{\Delta D_{j}=\Delta D_{i}}\right\}-\log \left\{p\left[\Delta D_{j}\right]_{\Delta D_{j}=\Delta D_{i}}\right\}
$$

The first term on the right is the updated probability evaluated at the discrete bin that matches the observed erosion volume for the $i^{t h}$ case. If the updated probability is higher than the prior probability 


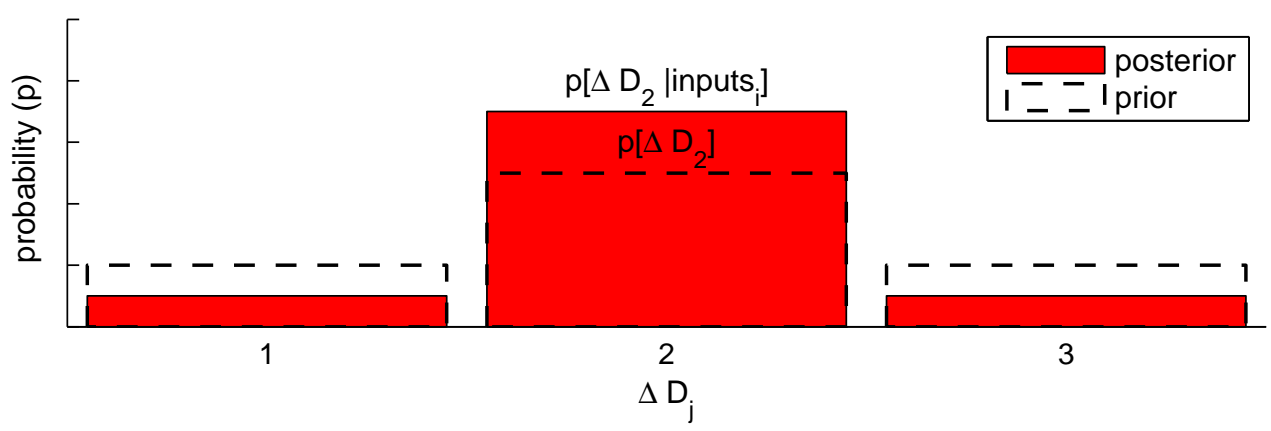

Figure 8: Given $\Delta D_{2}=\Delta D_{i}$ this figure shows the prior probability of a value in bin $\Delta D_{2}$ together with the posterior probability (after update with evidence inputs $s_{i}$ ) of the same bin. The posterior probability is higher than the prior probability, the log-likelihood formulated in Equation 8 is positive, so one is more certain about the outcome.

for the observed value, then the prediction is improved compared to the prior and the log-likelihood ratio is greater than zero. This indicates that the updated probability distribution is both different compared to the prior distribution and more accurate. On the other hand, if the updated probability of the observed value is lower than the prior probability, it indicates that the updated probability is either more uncertain than the prior or it is more confident but actually wrong.

Thus, the likelihood ratio scores the ability of the Bayesian Network to make skilful estimates of both mean value and uncertainty. A summation of the log-likelihood ratio over all observed cases provides a measure of how much better (or worse) the Bayesian Network prediction performed over the entire data set.

\section{RESULTS}

Once the Bayesian network is constructed and trained, it can be used for prediction by updating the prior probabilities (black dashed lines in Figure 9) of the input variables with data. Some face validity checks as well as the prediction skill and log-likelihood ratio of the network are dealt with in this section.

In the first face validity check, shown in Figure 9, the area, being the island Schiermonnikoog in the Wadden area, has been selected. In general, the Wadden area is characterised by small grain sizes, mild sloped profiles, wide beaches and low dunes. All four features are visible in the posterior distributions. In addition, the posterior distributions show that the profile volumes are relatively high and the erosion volumes are in the lower regions. In the network, the hydraulic conditions are not directly connected to the area. This means that no influence of the area choice is expected on the hydraulic conditions nodes, which is confirmed by Figure 9 . The second validity check concerns the selection of a relatively high water level, shown in Figure 10. Again, because of the absence of a direct link between the area and profile information, now only the hydraulic variables and the erosion volume are influenced. Since a high water level $\left(W L_{t}\right)$ is likely to coincide with high waves and long wave periods, we see a tendency to the higher bins of both wave height $\left(H s i g_{t}\right)$ and wave period $\left(T p_{t}\right)$. In addition, we see a larger probability for higher erosion volumes, w.r.t. the prior distribution, which is in line with the expectations.

Erosion volumes at several locations along the Dutch coast were hindcasted with testcases obtained from the distributions underlying the dataset described in previous section. A testcase thus is a set of variables representing a scenario and serves as input for the Bayesian network in order to make a prediction for the erosion volume. Since DURos+ results are considered as observations in this paper, a comparison between observed and predicted erosion volume is possible.

Figure 11 shows a comparison between predicted and observed erosion volumes for the network which was trained on the Holland coast area and applied for prediction of other transects in the same area. Although the spread is considerable (not precise), the average agreement is fairly good (accurate), being represented by a skill of 0.88 and a $\log$-likelihood of $L R=19$. This means that the network is capable of predicting on average the erosion volumes fairly well, if the cases to predict are covered by the training dataset. The spread can be reduced by increasing the number of bins (categories) per 

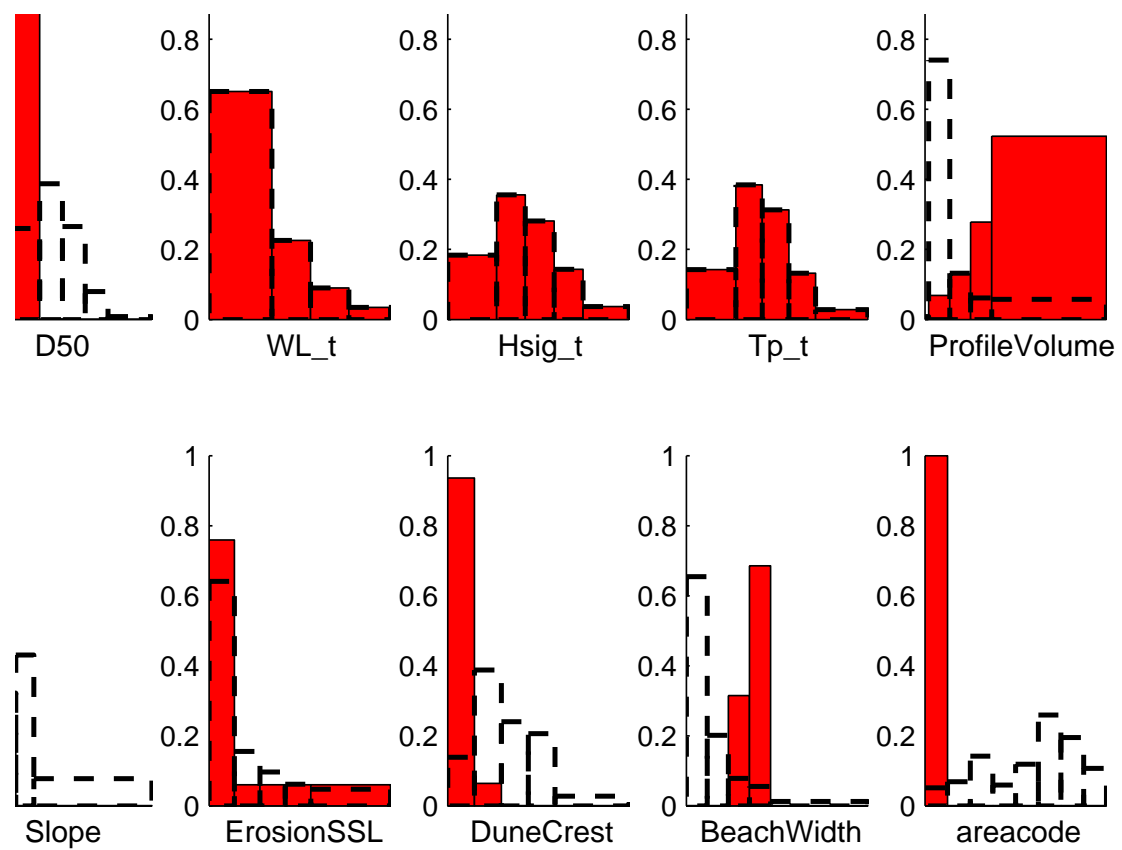

Figure 9: Prior (black dashed lines) and posterior probability distributions of Bayesian Network's variables. The island Schiermonnikoog, being part of the Wadden area, has been selected.

variable. Disadvantage of increasing the number of bins is that more data is needed to properly train the model.

In Figure 12 a similar predicted-observed comparison is made, for the same network that in this case was trained on the Wadden area data and used for prediction of the Holland coast. In this example, there is a deviation between both the profile indicators as well as the boundary conditions for both areas. In this case of prediction based on training that does not include the Holland coast, the skill as well as the log-likelihood ratio are worse $(0.52$ and $L R=-12)$ respectively. The low skill indicates that the best estimate from the Bayesian Network did not match the DuRos+ predictions as well as they did in the Wadden test and that the predicted uncertainty from the Bayesian Network did not correspond to the actual prediction errors. Adding more training data to both improve the best prediction and include the actual uncertainty is required. 


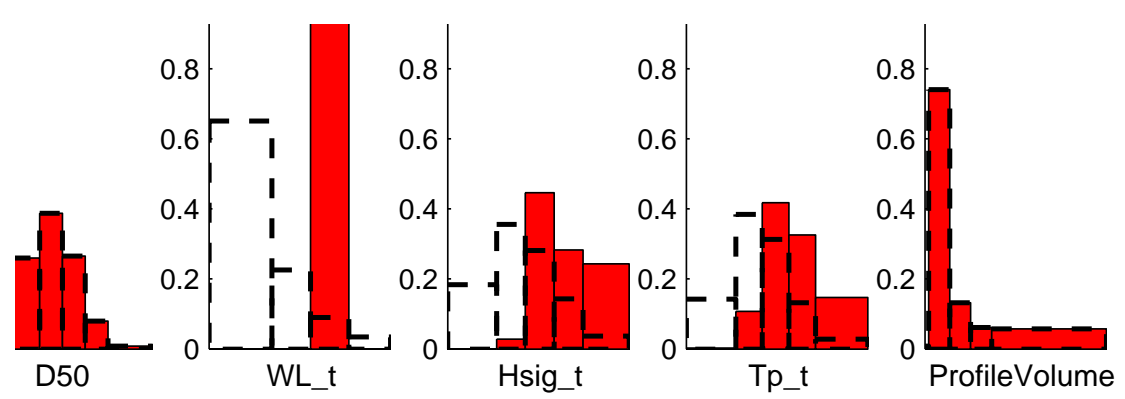

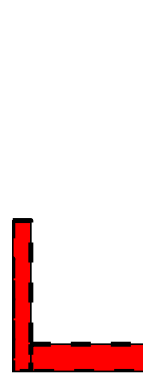

Slope
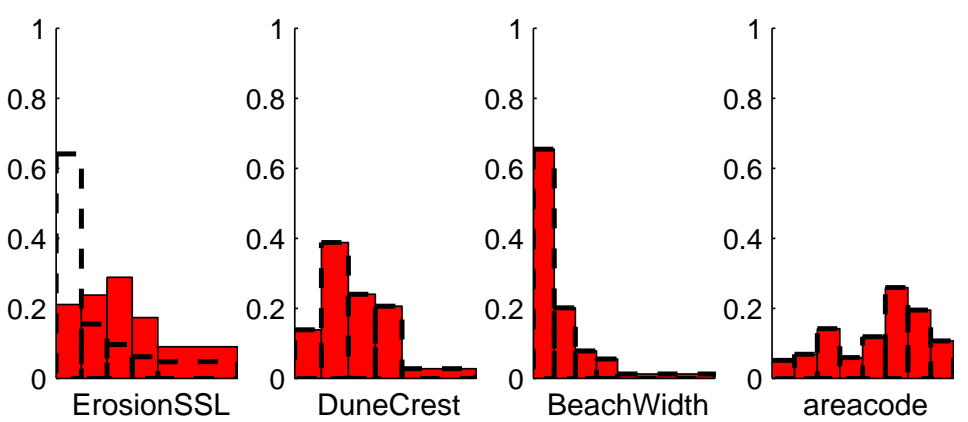

Figure 10: Prior (black dashed lines) and posterior probability distributions of Bayesian Network's variables. The water level bin around MSL+6 $\mathrm{m}$ has been selected.

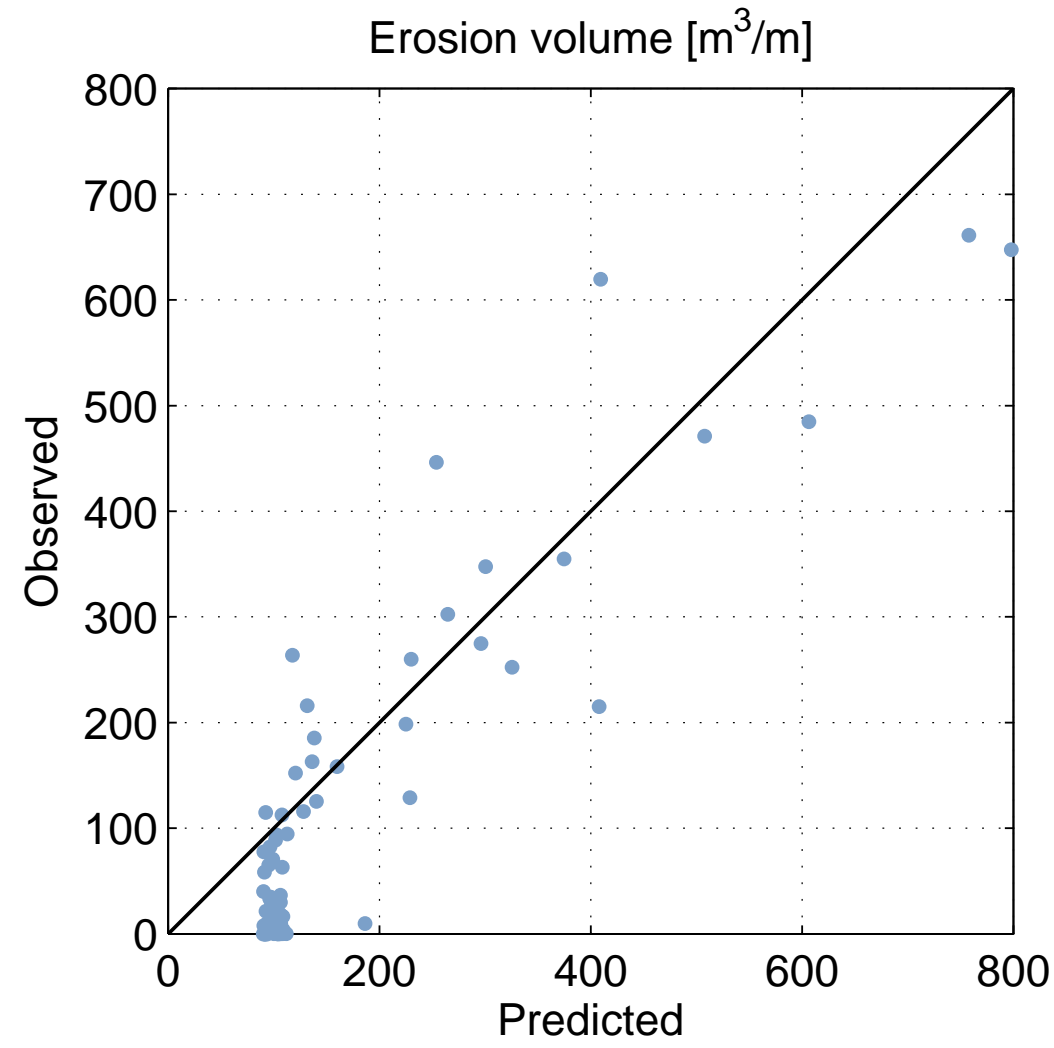

Figure 11: Observed vs. predicted for the Bayesian Network when trained on the Holland coast and applied on other transects in the same area. 


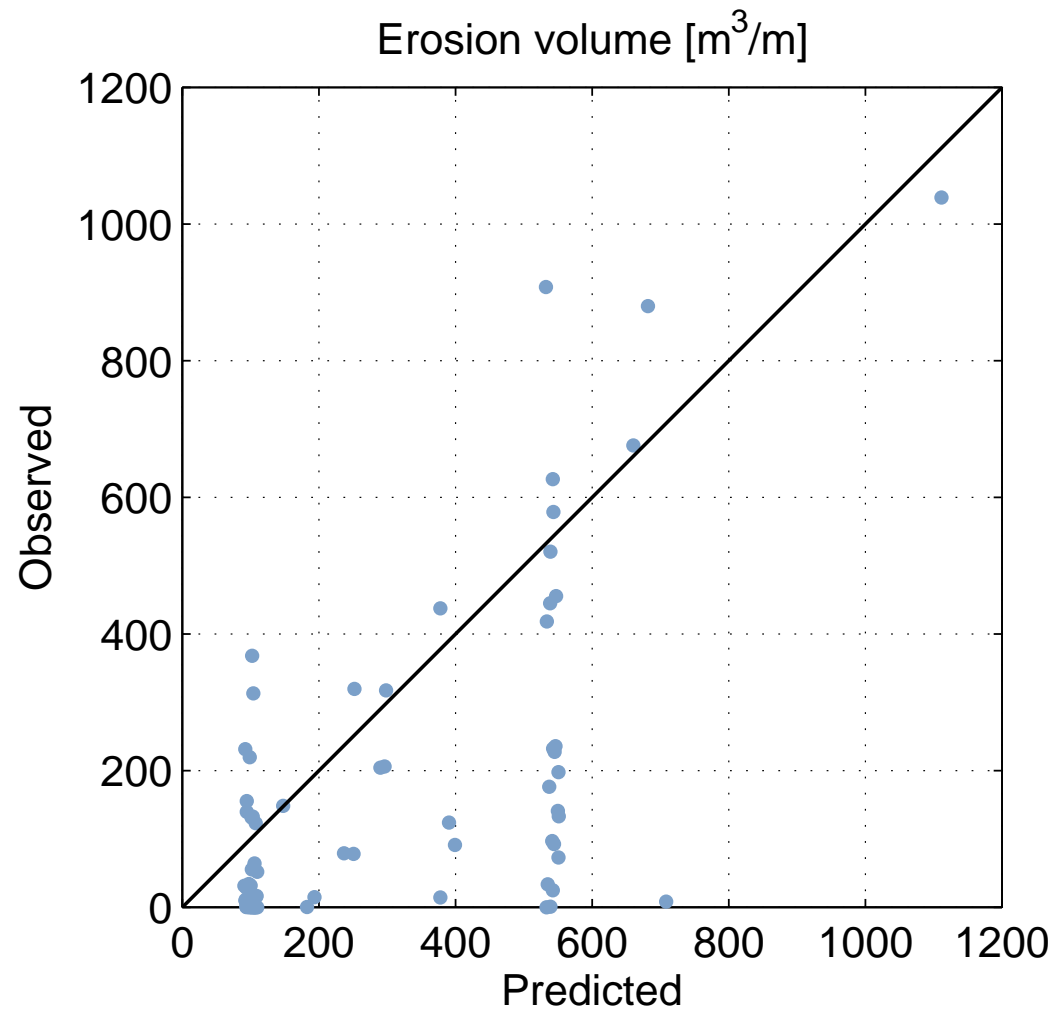

Figure 12: Observed vs. predicted for the Bayesian Network when trained on the Wadden coast and applied on the Holland coast transects. 


\section{CONCLUSIONS}

The applications of the Bayesian approach have demonstrated that Bayesian Networks are useful for quantifying the current state of prediction skill and uncertainty and identifying sensitivities of predictions to input uncertainties. We demonstrate that the Bayesian approach can (1) capture the dynamics of the numerical model accurately, and return $88 \%$ of the simulated variability of this model; (2) give fast predictions of dune erosion including the confidence interval; (3) deal with heterogeneous and missing data.

At the same time, it should be noted that these favourable conclusions are only possible if the cases to be predicted are well represented within the training data ranges. A Bayesian Network is only capable of interpolating and not extrapolating its training data. In addition, the precision of the results in this paper is not high (considerable spreading). This can be improved by increasing the number of bins per variable, implying that more training data is required.

\section{ACKNOWLEDGEMENTS}

Deltares, U.S. Geological Survey, Delft University of Technology, Technology Foundation STW and Rijkswaterstaat Waterdienst are gratefully acknowledged for their support of the work as summarized in this paper.

\section{REFERENCES}

Charniak, E. (1991). Bayesian networks without tears: making bayesian networks more accessible to the probabilistically unsophisticated. AI Mag., 12(4):50-63.

Cooper, G. F. (1990). The Computational Complexity of Probabilistic Inference Using Bayesian Belief Networks. Artificial Intelligence, 42(2-3):393-405.

Den Heijer, C., Baart, F., and van Koningsveld, M. (2011). Assessment of dune failure along the Dutch coast using a fully probabilistic approach. Geomorphology, 143-144:95-103.

Fishman, G. S. (1996). Monte Carlo: concepts, algorithms, and applications. Springer, New York. 698 pp.

Hasofer, A. M. and Lind, N. C. (1974). Exact and invariant second-moment code format. Journal of the Engineering Mechanics Division, ASCE, 100(EM1):111-121.

McCall, R. T., Van Thiel de Vries, J. S. M., Plant, N. G., Van Dongeren, A. R., Roelvink, J. A., Thompson, D. M., and Reniers, A. J. H. M. (2010). Two-dimensional time dependent hurricane overwash and erosion modeling at Santa Rosa Island. Coastal Engineering, 57(7):668-683.

Pearl, J. (1988). Probabilistic reasoning in intelligent systems: networks of plausible inference. Morgan Kaufmann Publishers Inc., San Francisco, CA, USA.

Plant, N. G. and Holland, K. T. (2011a). Prediction and assimilation of surf-zone processes using a Bayesian network: Part I: Forward models. Coastal Engineering, 58(1):119-130.

Plant, N. G. and Holland, K. T. (2011b). Prediction and assimilation of surf-zone processes using a Bayesian network: Part II: Inverse models. Coastal Engineering, 58(3):256-266.

Plant, N. G. and Stockdon, H. F. (2012). Probabilistic prediction of barrier-island response to hurricanes. Journal of Geophysical Research.

Roelvink, D., Reniers, A., van Dongeren, A., van Thiel de Vries, J., McCall, R., and Lescinski, J. (2009). Modelling storm impacts on beaches, dunes and barrier islands. Coastal Engineering, 56(11-12):1133-1152.

Van Dantzig, D. (1956). Economic decision problems for flood prevention. Econometrica, 24(3):276287.

Van Gent, M. R. A., van Thiel de Vries, J. S. M., Coeveld, E. M., de Vroeg, J. H., and van de Graaff, J. (2008). Large-scale dune erosion tests to study the influence of wave periods. Coastal Engineering, 55(12):1041-1051.

Vrijling, J. K. (2001). Probabilistic design of water defense systems in The Netherlands. Reliability Engineering $\mathcal{E}$ System Safety, 74(3):337-344. 
Wikle, C. K. and Berliner, L. M. (2007). A Bayesian tutorial for data assimilation. Physica D: Nonlinear Phenomena, 230(1-2):1-16. cited By (since 1996) 35.

WL | Delft Hydraulics (2007). Dune erosion; Product 3: Probabilistic dune erosion prediction method. Technical Report H4357 / A1414, Alkyon Hydraulic Consultancy \& Research / WL | Delft Hydraulics / Delft University of Technology. 